\title{
Diferenciação de Espécies daninhas Aguáticas pela Análise Multivariada de Caracteres Estruturais Foliares ${ }^{1}$
}

\author{
Differentiation of Aquatic Weeds by Multivariate Analysis of Foliar Structural Characters
}

\author{
COSTA, N.V. ${ }^{2}$, RODELLA, R.A. ${ }^{3}$ e MARTINS, D. ${ }^{4}$
}

\begin{abstract}
RESUMO - Algumas espécies de plantas aquáticas têm-se tornado problemáticas em reservatórios hidrelétricos no Brasil, devido a sua grande capacidade de reprodução. O objetivo deste trabalho foi diferenciar Brachiaria mutica, Brachiaria subquadripara, Panicum repens, Eichhornia crassipes, Heteranthera reniformis, Typha subulata e Enhydra anagallis, utilizandose 19 caracteres estruturais quantitativos do limbo foliar, que se relacionassem com a penetração e translocação de herbicidas. Amostras do terço médio do limbo foram fixadas em FAA 50, cortadas transversalmente em micrótomo com $8 \mathrm{Pm}$ de espessura e coradas com azul-detoluidina. Foram quantificados (\%) os seguintes caracteres estruturais da nervura central (NC) e da região internervural (IN): epidermes adaxial e abaxial, feixe vascular, bainha do feixe vascular, esclerênquima, parênquima e lacunas do aerênquima, além da espessura da folha, do número de estômatos e do número de tricomas nas faces adaxial e abaxial. Os 19 caracteres estruturais foram submetidos à Análise de Agrupamento e Análise de Componentes Principais. Houve a formação de três grupos principais: grupo $1-B$. mutica, B. subquadripara e P. repens (Poaceae); grupo 2 - E. crassipes e H. reniformis (Pontederiaceae) e E. anagallis (Asteraceae); e grupo 3 -apenas $T$. subulata (Typhaceae). Os caracteres com maior poder discriminatório foram: porcentagem de epiderme adaxial (IN); porcentagem de epiderme abaxial; feixe vascular; bainha do feixe vascular; esclerênquima e lacunas do aerênquima (NC e IN); espessura da folha e número de estômatos das faces adaxial e abaxial. Concluiu-se que os caracteres estruturais quantitativos permitiram diferenciar essas espécies daninhas aquáticas em fase vegetativa.
\end{abstract}

Palavras-chave: planta aquática, infestação, anatomia foliar.

\begin{abstract}
Some aquatic plant species have caused great damage to hydroelectric power dams in Brazil, due to their great capacity of reproduction. Nineteen quantitative structural characters of leaf blades were used to differentiate Brachiaria mutica, Brachiaria subquadripara, Panicum repens, Eichhornia crassipes, Heteranthera reniformis, Typha subulata, and Enhydra anagallis. Leaf blade samples were collected, fixed in FAA 50, infiltrated in glycol metacrylate resin, cut transversally with 8 Pm thickness, and stained with toluidine blue. Structural characters in vein and internerval regions were quantitatively evaluated (\%) for adaxial and abaxial epidermis, vascular bundle, vascular bundle sheath, esclerenchyma, parenchyma, and spongy aerenchyma. Leaf thickness, stomata and trichome number were determined at the internerval region. The quantitative structural characters of the leaf were submitted to Clusters Analysis and Principal Components Analysis. Three groups were formed according to similarity levels: group 1 - B. mutica, B. subquadripara, and P. repens (Poaceae); group 2 - E. crassipes and $\boldsymbol{H}$. reniformis (Pontederiaceae), and $\mathbf{E}$. anagallis (Asteraceae); group 3 - only for T. subulata (Typhaceae). The structural characters with high discriminatory degree were: adaxial epidermis (internerval region); abaxial epidermis, vascular bundle, vascular bundle sheath, esclerenchyma and spongy aerenchyma (middle vein and internerval region); leaf thickness; and stomata number. The quantitative structural characters allowed the differentiation of these aquatic weed species during the vegetative period.
\end{abstract}

Keywords: aquatic plant, infestation, leaf anatomy.

1 Recebido para publicação em 29.8.2005 e na forma revisada em 24.2.2006.

2 Eng.-Agr., aluno de Pós-Graduação, Dep. Produção Vegetal-Agricultura, FCA-UNESP, <neumarcio@ fca.unesp.br>; ${ }^{3}$ Prof. Dr., Dep. de Botânica, Instituto de Biociências de Botucatu-UNESP; ${ }^{4}$ Prof. Dr., Dep. de Produção Vegetal-Agricultura, FCA-UNESP. Caixa Postal 237, 18603-970 Botucatu-SP.

Planta Daninha, Viçosa-MG, v. 24, n. 1, p. 13-20, 2006 


\section{INTRODUÇÃO}

Nos ecossistemas aquáticos, as plantas são a principal fonte produtora de biomassa (Esteves, 1998). O excessivo desenvolvimento dessas plantas pode ocasionar vários inconvenientes no que se refere ao transporte hidroviário, à pesca, à produção de energia, ao abastecimento de água, aos esportes náuticos, à proliferação de vetores de doenças, entre outros. Algumas espécies de crescimento rápido podem suprimir outras menos agressivas, desejáveis para manutenção da diversidade, ou modificar negativamente algumas características físicas da água (Cardoso et al., 2002).

Em levantamento nos reservatórios operados pela Companhia Energética de São Paulo-CESP (Três Irmãos, Jupiá, Ilha Solteira, Porto Primavera, Paraibuna e Jaguari), Tanaka et al. (2002) identificaram 29 espécies de plantas aquáticas, distribuídas em 17 famílias, dentre as quais Brachiaria mutica, Brachiaria subquadripara, Enhydra anagallis, Heteranthera reniformis, Panicum repens, Typha subulata e Eichhornia crassipes têm se tornado problemáticas, devido à sua grande capacidade reprodutiva e produção de biomassa.

Príncipe et al. (1997) afirmam que um dos principais transtornos ocasionados pela grande incidência de plantas aquáticas, notadamente em épocas de cheias, está relacionado com a redução da potência das unidades geradoras de energia das usinas hidrelétricas, pois o acúmulo de detritos vegetais nas grades da tomada de água de uma unidade geradora impede que esta gere sua potência máxima, reduzindo assim a capacidade de produção de energia da usina.

O controle químico apresenta-se altamente seletivo às plantas daninhas aquáticas, além de evitar danos à fauna, como ocorre no controle mecânico, pela coleta indesejada de insetos e alevinos. O uso de herbicidas apresenta-se como excelente alternativa de manejo, promovendo resultados rápidos de controle, além de manter a população de plantas daninhas em níveis satisfatórios, sem prejuízos para o uso da água (Martins et al., 2002).

A eficiência do controle químico está relacionada a diversos fatores, sendo muito importante o conhecimento das características estruturais da planta. Hess \& Falk (1990) ressaltam a diversidade morfológica da epiderme foliar, em várias espécies, indicando que topografia, grau e tipo de cera epicuticular, tricomas e estômatos influenciam a deposição dos herbicidas sobre a superfície da folha. As características estruturais possibilitam ainda diferenciar espécies. Entretanto, estudos sobre anatomia foliar de plantas aquáticas relacionados com a deposição, absorção e translocação de herbicidas são bastante escassos no Brasil.

De acordo com Procópio et al. (2003b), a morfologia das folhas influencia a quantidade de produto interceptado e retido, porém é a anatomia foliar que determina a facilidade com que serão absorvidos. Assim, o estudo da anatomia foliar de plantas daninhas destacase como uma técnica importante na identificação e descrição de estruturas que podem influenciar a absorção dos herbicidas, além de auxiliar na diferenciação de espécies.

Ferreira et al. (2002a, b) e Procópio et al. (2003b) relatam que o estudo anatômico foliar pode melhorar o entendimento sobre as barreiras que cada espécie impõe à penetração dos herbicidas e subsidiar a busca de estratégias para superá-las. Dessa maneira, a análise da estrutura foliar pode permitir a identificação de espécies suscetiveis, tolerantes ou resistentes a determinado produto químico, definindo sua seletividade.

O objetivo deste trabalho foi diferenciar sete espécies de plantas daninhas aquáticas, ocorrentes em reservatórios hidrelétricos do Estado de São Paulo, utilizando-se 19 caracteres estruturais quantitativos do limbo foliar, relacionados à penetração e translocação de herbicidas, visando contribuir para o sucesso do controle químico de plantas daninhas.

\section{MATERIAL E MÉTODOS}

As espécies de plantas daninhas aquáticas utilizadas foram: Brachiaria mutica, Brachiaria subquadripara, Eichhornia crassipes, Enhydra anagallis, Heteranthera reniformis, Panicum repens e Typha subulata.

O experimento foi conduzido no Núcleo de Pesquisas Avançadas em Matologia (NUPAM), do Departamento de Produção Vegetal da 
Faculdade de Ciências Agronômicas de Botucatu - UNESP. As plantas daninhas aquáticas foram reproduzidas em pleno sol, por meio de suas estruturas de dispersão vegetativa, e acondicionadas em caixas-d'água de fibra de vidro de 320 litros, contendo no fundo uma camada de $15 \mathrm{~cm}$ de solo. A análise química do solo apresentou os seguintes valores: $\mathrm{pH}=5 \mathrm{em} \mathrm{CaCl}_{2}$; matéria orgânica $=16 \mathrm{~g} \mathrm{dm}^{-3}$; $\mathrm{CTC}=75 / \mathrm{P}=70 \mathrm{mg} \mathrm{dm}^{-3} ; \mathrm{K}=1,9 / \mathrm{Ca}=28$ $/ \mathrm{Mg}=9 \mathrm{mmol}_{\mathrm{c}} \mathrm{dm}^{-3}$; e saturação por bases = $39 \%$.

As plantas foram avaliadas antes do florescimento, quando se encontravam totalmente desenvolvidas, amostrando-se quatro folhas, e cada uma representou uma repetição. A folha foi escolhida de acordo com o hábito de crescimento de cada espécie de planta daninha aquática. Para B. mutica, B. subquadripara, $P$. repens, $H$. reniformis e $E$. anagallis coletouse a terceira folha totalmente expandida, contada a partir do ápice. Em se tratando de E. crassipes, foi coletada a folha mais próxima do centro da roseta. Para T. subulata coletouse a terceira folha totalmente expandida, contada a partir da primeira folha com bainha, no sentido do centro da roseta para fora.

Foram tomadas porções do terço médio do limbo foliar, compreendendo a nervura central e a região internervural, fixando-as em FAA 50 (formaldeído + ácido acético glacial + álcool $50 \%$ ) durante 48 horas, sendo, a seguir, conservadas em álcool 70\% (Johansen, 1940).

As amostras foliares foram infiltradas com historresina (Gerrits, 1991), cortadas transversalmente em micrótomo rotatório com $8 \mu \mathrm{m}$ de espessura e coradas com azul-de-toluidina 0,05\% (O’Brien et al., 1964). Posteriormente, foram feitas observações microscópicas das estruturas da nervura central e da região internervural do limbo, e os limites e contornos dos tecidos, desenhados com o auxílio de microscópio de projeção; as áreas foram mensuradas em mesa digitalizadora acoplada a um computador equipado com o programa de Sistema de Planimetria (SPLAN), desenvolvido pelo CINAG - UNESP de Botucatu/SP (Souza et al., 2005).

Nas estruturas presentes no limbo, foram quantificados caracteres anatômicos da nervura central e da região internervural (expressos em percentual), referentes a epiderme das faces adaxial e abaxial, feixe vascular, bainha do feixe vascular (endoderme), esclerênquima, parênquima e lacunas do aerênquima. Foi também mensurada, na região internervural, a espessura do limbo foliar.

O número de estômatos e de tricomas por milímetro quadrado da superfície foliar foi determinado nas faces adaxial e abaxial do limbo, utilizando-se a técnica de impressão da epiderme em adesivo à base de éster de cianoacrilato (Souza et al., 2005), com 16 repetições por face em cada espécie. A média do número de estômatos e de tricomas, em cada impressão da epiderme, foi originária da contagem de cinco campos de observação, tomados ao acaso, totalizando 80 campos de amostragem por face foliar.

Os 19 caracteres estruturais quantitativos do limbo foliar foram submetidos aos testes estatísticos multivariados de Análise de Agrupamento e Análise de Componentes Principais (Sneath \& Sokal, 1973), a fim de agrupar as espécies de acordo com seu grau de similaridade e verificar a capacidade discriminatória dos caracteres originais no processo de formação dos agrupamentos.

\section{RESULTADOS E DISCUSSÃO}

Os valores médios referentes aos 19 caracteres estruturais quantitativos do limbo foliar, para as sete espécies de plantas daninhas aquáticas, empregados na realização da análise estatística multivariada encontram-se na Tabela 1.

Na Análise de Agrupamento, representada na forma de dendrograma (Figura 1), foi possível agrupar as sete espécies estudadas de forma que aquelas pertencentes a um mesmo grupo apresentassem maior similaridade que as de grupos diferentes. Houve a formação de três grupos principais, em nível de 0,40 na escala de distância de similaridade, os quais apresentaram a seguinte disposição: grupo 1 - formado pelas três espécies de gramíneas (Poaceae): Brachiaria mutica, B. subquadripara e Panicum repens; grupo 2 - constituído pelas espécies de Pontederiaceae, Eichhornia crassipes e Heteranthera reniformis, e pela espécie de Asteraceae, Enhydra anagallis; grupo 3 - formado somente por Typha subulata,

Planta Daninha, Viçosa-MG, v. 24, n. 1, p. 13-20, 2006 
Tabela 1 - Valores médios de 19 caracteres estruturais quantitativos do limbo foliar de sete espécies de plantas daninhas aquáticas. Botucatu-SP, 2004

\begin{tabular}{|c|c|c|c|c|c|c|c|}
\hline Caráter & ENYAN & PANPU & BRASU & PANRE & EICCR & HETRE & TYPSU \\
\hline & \multicolumn{7}{|c|}{ Região da Nervura Central } \\
\hline$\%$ epiderme face adaxial & 2,97 & 4,69 & 14,68 & 6,85 & 6,41 & 5,44 & 0,42 \\
\hline$\%$ epiderme face abaxial & 3,92 & 5,73 & 5,84 & 6,18 & 4,25 & 6,30 & 0,41 \\
\hline$\%$ feixe vascular & 6,14 & 10,73 & 7,79 & 9,94 & 4,20 & 5,08 & 3,34 \\
\hline$\%$ bainha feixe vascular (endoderme) & 3,02 & 20,31 & 27,27 & 22,49 & 13,00 & 11,15 & 0 \\
\hline$\%$ esclerênquima & 1,64 & 5,52 & 2,08 & 3,57 & 1,12 & 1,22 & 0,40 \\
\hline \% parênquima & 82,31 & 53,02 & 42,34 & 50,97 & 57,98 & 52,04 & 28,19 \\
\hline \multirow{2}{*}{$\%$ lacunas do aerênquima } & 0 & 0 & 0 & 0 & 13,04 & 18,77 & 67,24 \\
\hline & \multicolumn{7}{|c|}{ Região Internervural } \\
\hline \% epiderme face adaxial & 4,76 & 14,49 & 14,89 & 11,07 & 6,36 & 6,43 & 0,44 \\
\hline$\%$ epiderme face abaxial & 4,63 & 6,63 & 6,99 & 9,56 & 4,10 & 6,50 & 0,48 \\
\hline$\%$ feixe vascular & 1,03 & 12,22 & 8,46 & 11,75 & 4,80 & 3,04 & 3,67 \\
\hline$\%$ bainha feixe vascular (endoderme) & 3,34 & 26,88 & 29,23 & 33,33 & 16,99 & 5,74 & 0 \\
\hline$\%$ esclerênquima & 0 & 2,97 & 1,65 & 2,19 & 1,89 & 0 & 0,42 \\
\hline$\%$ parênquima & 86,25 & 36,82 & 38,79 & 32,10 & 54,53 & 61,96 & 28,69 \\
\hline \% lacunas do aerênquima & 0 & 0 & 0 & 0 & 11,33 & 16,32 & 66,30 \\
\hline Espessura da folha $\left(\times 10^{2} \mathrm{~mm}\right)$ & 23,71 & 14,75 & 14,22 & 16,10 & 46,51 & 24,78 & 291,85 \\
\hline $\mathrm{N}^{\mathrm{o}}$ estômatos $\mathrm{mm}^{-2}$ (face adaxial) & 221,58 & 179,76 & 176,17 & 164,15 & 130,88 & 291,33 & 537,21 \\
\hline $\mathrm{N}^{\mathrm{o}}$ estômatos $\mathrm{mm}^{-2}$ (face abaxial) & 194,08 & 145,47 & 134,72 & 102,09 & 150,96 & 184,23 & 521,61 \\
\hline $\mathrm{N}^{\mathrm{o}}$ tricomas $\mathrm{mm}^{-2}$ (face adaxial) & 0 & 14,60 & 0,76 & 0,51 & 0 & 0 & 0 \\
\hline $\mathrm{N}^{\mathrm{o}}$ tricomas $\mathrm{mm}^{-2}$ (face abaxial) & 0 & 37,75 & 39,02 & 1,00 & 0 & 0 & 0 \\
\hline
\end{tabular}

ENYAN: Enhydra anagallis; PANPU: Brachiaria mutica; BRASU: Brachiaria subquadripara; PANRE: Panicum repens; EICCR: Eichhornia crassipes; HETRE: Heteranthera reniformis; TYPSU: Typha subulata.

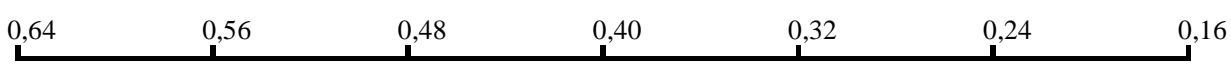

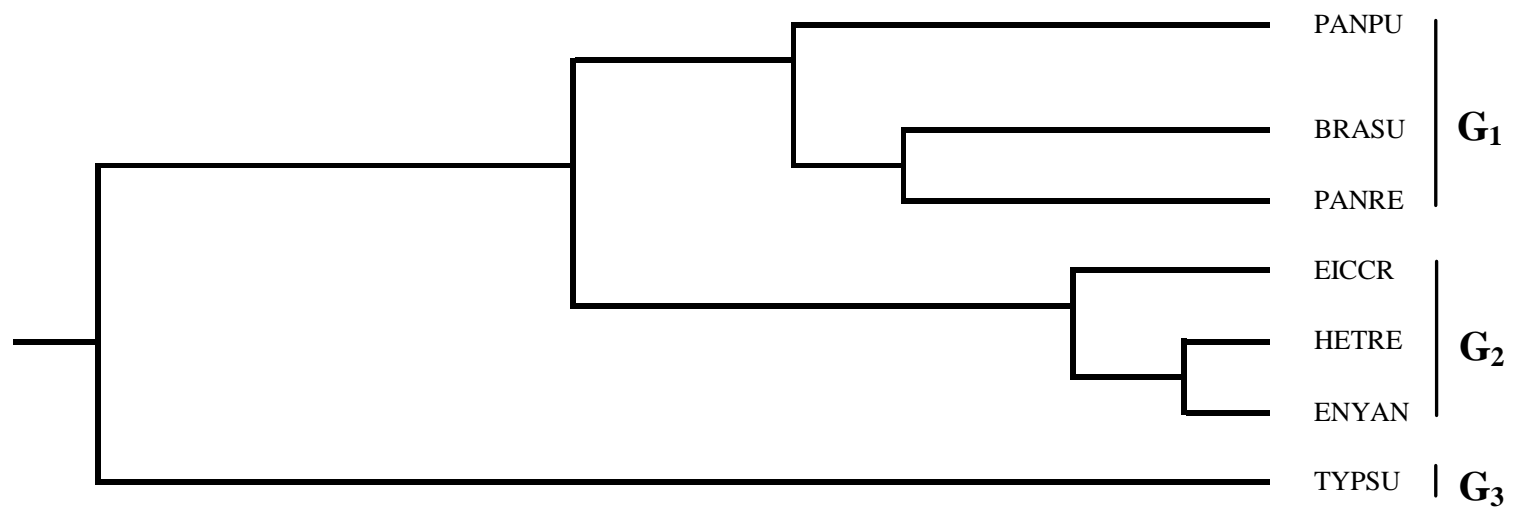

ENYAN: Enhydra anagallis; PANPU: Brachiaria mutica; BRASU: Brachiaria subquadripara; PANRE: Panicum repens; EICCR: Eichhornia crassipes; HETRE: Heteranthera reniformis; TYPSU: Typha subulata.

Figura 1 - Dendrograma resultante da Análise de Agrupamento dos caracteres estruturais quantitativos das folhas, utilizando-se a distância euclidiana média entre as espécies de plantas daninhas aquáticas.

Planta Daninha, Viçosa-MG, v. 24, n. 1, p. 13-20, 2006

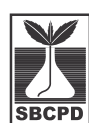


pertencente à família Typhaceae. Verificou-se também que as espécies do grupo 1 (Poaceae) e a do grupo 3 (Typhaceae) apresentaram maior dissimilaridade, enquanto as do grupo 2 (Pontederiaceae e Asteraceae) mostraram maior similaridade com base nos caracteres estruturais quantitativos avaliados.

Na Tabela 2 são apresentados os coeficientes de correlação entre os 19 caracteres estruturais do limbo foliar e os dois primeiros componentes principais $\left(\mathrm{Y}_{1}\right.$ e $\left.\mathrm{Y}_{2}\right)$. No conjunto, esses componentes foram responsáveis por $81,98 \%$ da informação acumulada pelos caracteres avaliados, constituindo-se em indicadores eficientes de dissimilaridade, comportando-se, portanto, como caracteres diferenciadores entre as espécies de daninhas aquáticas estudadas.

Para $Y_{1}$, as porcentagens de epiderme da face adaxial da região internervural e de bainha do feixe vascular (endoderme) da nervura central e da região internervural foram os caracteres que mais contribuíram para a discriminação das sete espécies estudadas, seguindo-se as porcentagens de epiderme da face abaxial, feixe vascular, esclerênquima e lacunas de aerênquima da nervura central e da região internervural, bem como a espessura da folha, do número de estômatos das faces adaxial e abaxial e de tricomas da face abaxial (Tabela 2). Este componente $\left(Y_{1}\right)$ apresentou $63,58 \%$ da informação retida, a qual é considerada satisfatória para explicar a contribuição dos caracteres na formação dos agrupamentos.

Para $Y_{2}$, a informação retida foi de $18,40 \%$, que é um valor considerado baixo; conseqüentemente, apenas o caráter relacionado com a porcentagem de parênquima da nervura central e da região internervural apresentou poder de discriminação satisfatório.

A dispersão gráfica (Figura 2), resultante da Análise de Componentes Principais, também mostrou a formação de três grupos, com relação às sete espécies estudadas, revelando haver boa concordância entre esses resultados e aqueles obtidos na Análise de Agrupamento (Figura 1).

Na análise conjunta de $Y_{1}$ e da dispersão gráfica, as espécies de Poaceae, ou seja, as gramíneas constituintes do grupo 1, apresentaram os maiores valores dos caracteres relativos a epiderme, feixe vascular, bainha do feixe vascular (endoderme) e esclerênquima da nervura central e da região internervural, bem como os menores valores dos caracteres referentes a número de estômatos, espessura da folha e lacunas de aerênquima (inexistentes no limbo foliar de gramíneas) e maior valor do número de tricomas (Tabela 1). Resultados contrários foram constatados para a espécie constituinte do grupo 3 (Typhaceae), enquanto as do grupo 2 (Pontederiaceae e Asteraceae) mostraram tendência em apresentar valores intermediários.

Em trabalho semelhante, Rocha (2001) avaliou diferenciações anatômicas em Commelinaceae pela espessura da folha, pelo tamanho da nervura central e pela quantidade de feixes vasculares presentes no caule; os resultados mostraram maiores valores para suscetiveis a herbicidas $(C$. benghalensis $\mathrm{e}$ C. villosa) e menores para tolerantes (C. diffusa e C. erecta), o que explica a facilidade ou dificuldade de translocação.

Tabela 2 - Coeficientes de correlação entre os 19 caracteres estruturais quantitativos do limbo foliar de sete espécies de plantas daninhas aquáticas e os dois primeiros componentes principais ( $\mathrm{Y}_{1}$ e $\mathrm{Y}_{2}$ ). Botucatu-SP, 2004

\begin{tabular}{|c|c|c|}
\hline Caráter & $Y_{1}$ & $\mathrm{Y}_{2}$ \\
\hline$\%$ epiderme face adaxial (NC) & 0,6774 & 0,1410 \\
\hline$\%$ epiderme face abaxial (NC) & 0,8280 & 0,4127 \\
\hline$\%$ feixe vascular (NC) & 0,8835 & $-0,1503$ \\
\hline$\%$ bainha feixe vascular (endoderme) (NC) & 0,9271 & $-0,0880$ \\
\hline \% esclerênquima (NC) & 0,8177 & $-0,2751$ \\
\hline \% parênquima $(\mathrm{NC})$ & 0,1515 & 0,7858 \\
\hline \% lacunas do aerênquima (NC) & $-0,8343$ & $-0,5108$ \\
\hline$\%$ epiderme face adaxial (RIN) & 0,9764 & $-0,1065$ \\
\hline \% epiderme face abaxial (RIN) & 0,8437 & 0,3138 \\
\hline$\%$ feixe vascular (RIN) & 0,8163 & $-0,4820$ \\
\hline \% bainha feixe vascular (endoderme) (RIN) & 0,9178 & $\begin{array}{l}-0,1705 \\
-0,1705\end{array}$ \\
\hline \% esclerênquima (RIN) & 0,7846 & $-0,4392$ \\
\hline$\%$ parênquima (RIN) & $-0,2222$ & 0,8497 \\
\hline \% lacunas do aerênquima (RIN) & $-0,8279$ & $-0,5289$ \\
\hline Espessura da folha (RIN) & $-0,7866$ & $-0,5969$ \\
\hline $\mathrm{N}^{0}$ estômatos mm $\mathrm{m}^{-2}$ (face adaxial) & $\begin{array}{ll}-0,8116 \\
-2\end{array}$ & $-0,4648$ \\
\hline $\mathrm{N}$ - estômatos mm $\mathrm{mm}^{-2}$ (face abaxial) & $\begin{array}{ll}-0,8396 \\
-2\end{array}$ & $-0,5214$ \\
\hline $\mathrm{N}^{0}$ tricomas $\mathrm{mm}^{-2}$ (face adaxial) & 0,5513 & $-0,4992$ \\
\hline $\mathrm{N}^{\mathrm{o}}$ tricomas $\mathrm{mm}^{-2}$ (face abaxial) & 0,7032 & $-0,4356$ \\
\hline$\%$ informação retida & 63,58 & 18,40 \\
\hline \% informação acumulada & 63,58 & 81,98 \\
\hline
\end{tabular}

NC: nervura central. RIN: região internervural.

Planta Daninha, Viçosa-MG, v. 24, n. 1, p. 13-20, 2006 


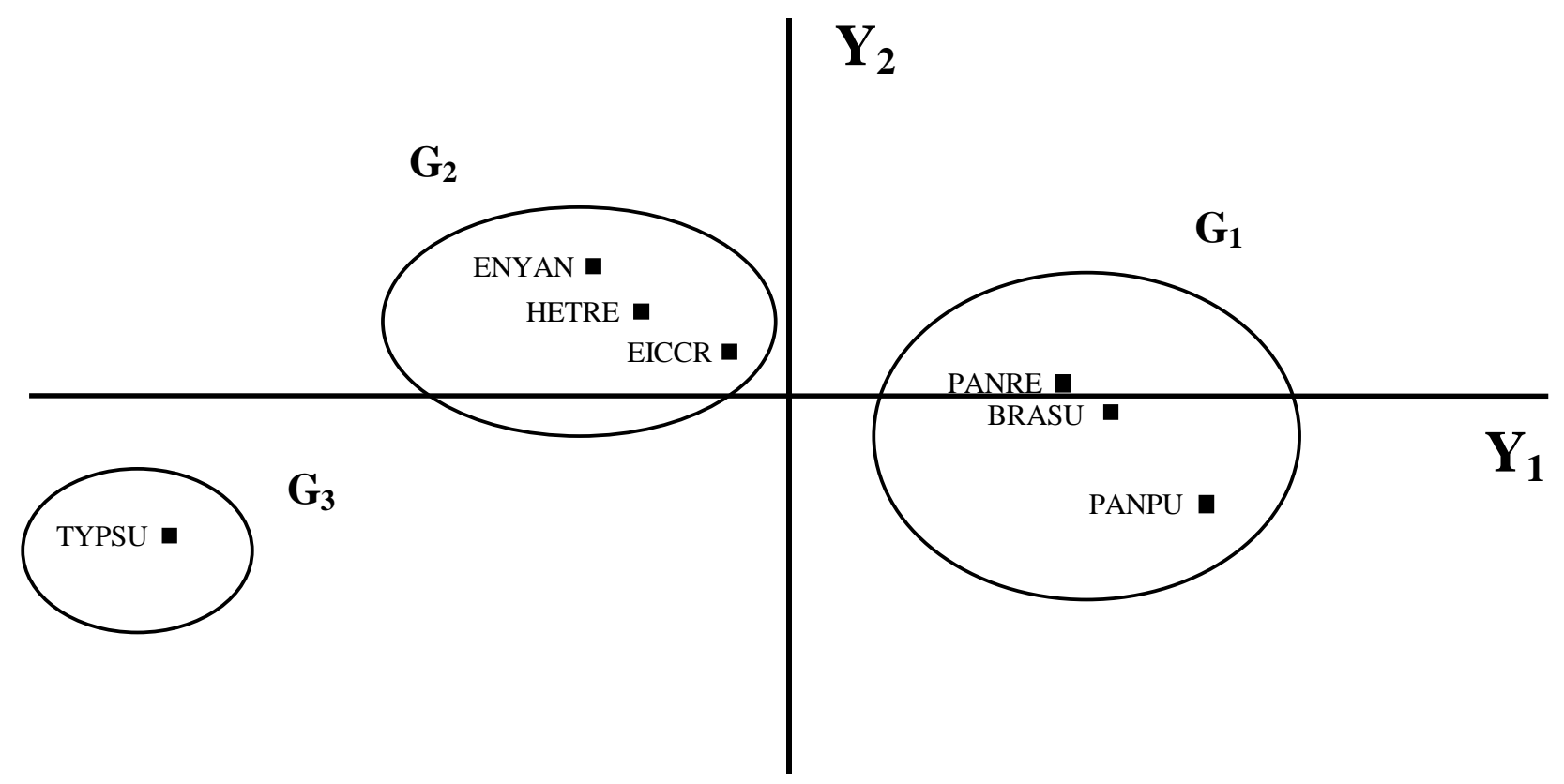

ENYAN: Enhydra anagallis; PANPU: Brachiaria mutica; BRASU: Brachiaria subquadripara; PANRE: Panicum repens; EICCR: Eichhornia crassipes; HETRE: Heteranthera reniformis; TYPSU: Typha subulata.

Figura 2 - Representação gráfica da dispersão das espécies de plantas daninhas aquáticas, utilizando-se os dois primeiros componentes principais $\left(\mathrm{Y}_{1}\right.$ e $\left.\mathrm{Y}_{2}\right)$, para o conjunto dos 19 caracteres estruturais quantitativos da folha.

Procópio et al. (2003a) evidenciam como principal barreira à penetração dos herbicidas nas folhas a ocorrência de maior espessura da cutícula e da epiderme da face adaxial, alto teor de cera epicuticular, alta densidade de tricomas, baixa densidade de estômatos e alto teor de cera epicuticular.

Com base nos maiores valores dos caracteres estruturais quantitativos de epiderme, feixe vascular, bainha do feixe vascular (endoderme), esclerênquima e número de tricomas, bem como nos menores valores para número de estômatos e espessura da folha, pode-se inferir que as espécies do grupo 1 (Poaceae) apresentam maior dificuldade de controle, seguida das espécies do grupo 2 (Pontederiaceae e Asteraceae) e do grupo 3 (Typhaceae). Martins et al. (2002), Carbonari et al. (2003) e Silva (2003) constataram também que $B$. mutica e $B$. subquadripara (Poaceae) mostraram maior dificuldade de controle do que $T$. subulata (Typhaceae) e E. crassipes (Pontederiaceae) quando se utilizou glyphosate $\left(7 \mathrm{~L} \mathrm{ha}^{-1}\right)$ com adjuvantes.
Buick et al. (1993) consideram os estômatos uma das principais vias de penetração de herbicidas. Dessa forma, as espécies de gramíneas estudadas, por possuírem menor número de estômatos no limbo foliar, podem apresentar maior dificuldade de controle que as demais. Entretanto, Greene \& Bukovac (1974) verificaram que a penetração pelos estômatos não se caracterizou como uma importante rota de entrada de soluções aquosas com tensão superficial próxima à da água. Contudo, a redução da tensão superficial pela adição de surfatantes pode induzir o aumento da absorção estomática.

De acordo com Hess \& Falk (1990) e Procópio et al. (2003b), a presença de tricomas, mesmo em pequenas quantidades na superfície foliar, pode interceptar e causar a aderência das gotas de pulverização, impedindo o seu contato com as células da epiderme e reduzindo a eficiência dos herbicidas. Das espécies estudadas, somente as gramíneas apresentaram tricomas, característica esta que pode contribuir para a sua maior dificuldade 
de controle. No entanto, a eficiência da absorção de herbicidas pelos tricomas e a translocação destes para as células da epiderme são ainda pouco conhecidas (Hess \& Falk, 1990).

Assim, as estruturas anatômicas foliares podem influenciar a deposição, retenção, absorção e translocação de soluções aplicadas sobre as folhas, funcionando como barreiras, que podem estar relacionadas com a maior ou menor resistência de determinada planta daninha aos herbicidas. Contudo, pouco se conhece sobre a proporção da contribuição de cada caráter estrutural do limbo foliar no processo de absorção e translocação de herbicidas.

Verificou-se a ocorrência de similaridades entre Poaceae e Typhaceae, ambas pertencentes à subclasse Commelinidae, da classe Liliopsida (monocotiledôneas), bem como a existência de similaridades entre Pontederiaceae, da subclasse Liliidae, também pertencente à classe Liliopsida (monocotiledôneas), e Asteraceae, da subclasse Asteridae, da classe Magnolipsida (dicotiledôneas).

Assim, a porcentagem de parênquima do limbo foliar permitiu discriminar as espécies de Commelinidae das espécies de Liliidae e Asteridae, que, segundo Cronquist (1981), são consideradas as subclasses mais evoluídas, respectivamente, nas classes Liliopsida e Magnoliopsida.

As diferenças estruturais encontradas nas espécies daninhas aquáticas podem auxiliar na correta identificação dessas espécies em levantamentos realizados em reservatórios hidrelétricos, bem como nas tomadas de decisão para o manejo químico.

\section{LITERATURA CITADA}

BUICK, R. D.; BUCHAN, G. D.; FIELD, R. J. The role of surface tension of spreading droplets in absorption of a herbicide formulation via leaf stomata. Pest. Sci., v. 38, p. 227-235, 1993.

CARBONARI, C. A.; MARTINS, D.; TERRA, M. A.

Controle de Brachiaria subquadripara e Brachiaria mutica através de diferentes herbicidas aplicados em pósemergência. Planta Daninha, v. 21, p. 79-84, 2003. Edição especial.
CARDOSO, L. R. et al. Variabilidade genética de acessos de aguapé coletados no Estado de São Paulo. Planta Daninha, v. 20, p. 1-6, 2002. Edição especial.

CRONQUIST, A. An integrated system of classification of flowering plants. New York: Columbia University, 1981. $1262 \mathrm{p}$.

ESTEVES, F. A. Fundamentos de limnologia. 2.ed. Rio de Janeiro: Interciência, 1998. 602 p.

FERREIRA, E. A. et al. Estudos anatômicos de folhas de plantas daninhas. I. Nicandra physaloides, Solanum americanum e Raphanus raphanistrum. Planta Daninha, v. 20 , n. 2, p. 159-167, 2002a.

FERREIRA, E. A. et al. Estudos anatômicos de folhas de plantas daninhas. II. Bidens pilosa, Emilia sonchifolia, Agerantum conyzoides e Sonchus asper. Planta Daninha, v. 20, n. 3 , p.327-335, 2002 b.

GERRITS, P. O. The application of glycol metacrylate in histotechnology: same fundamental principles. Germany: Leica Gmgh, 1991. 80 p.

GREENE, D. W.; BUKOVAC, M. J. Stomatal penetration: effect of surfactants and role in foliar absorption. Am. J. Botany, v. 61, n. 1, p. 100-106, 1974.

HESS, F. D.; FALK, R. H. Herbicide deposition on leaf surfaces. Weed Sci., v. 38, p. 280-288, 1990.

JOHANSEN, D. A. Plant microtechmique. New York: McGrow-Hill Book, 1940. 523 p.

MARTINS, D. et al. Controle químico de Pistia stratiotes, Eichhornia crassipes e Salvinia molesta em caixas d'água. Planta Daninha, v. 20, p. 83-88, 2002. Edição Especial

O’BRIEN, T. P.; FEDER, N.; McCULLY, M. E.

Polychromatic staining of plant cellular wall by toluidine blue. Protoplasma, v. 59, p. 368-373, 1964.

PRÍNCIPE, C. R.; KURATANI, H.; MELONI, M. L. B. Impactos da afluência de elódeas na operação e manutenção da usina hidroelétrica Eng. Souza Dias (Jupiá)-CESP. In: CONGRESSO BRASILEIRO DA CIÊNCIA DAS PLANTAS DANINHAS (Workshop de Plantas Aquáticas), 21., 1997, Caxambu, MG. Resumos... Caxambu: SBCPD, 1997. p. $5-8$.

PROCÓPIO, S. O. et al. Estudos anatômicos de folhas de plantas daninhas de grande ocorrência no Brasil. III. Galinsoga parviflora, Crotalaria incana, Conyza bonariensis e Ipomoea cairica. Planta Daninha, v. 21, n. 1, p. 1-9, 2003a.

PROCÓPIO, S. O. et al. Anatomia foliar de plantas daninhas do Brasil. Viçosa: UFV. 2003b, v. 1. 118 p.

Planta Daninha, Viçosa-MG, v. 24, n. 1, p. 13-20, 2006 
ROCHA, D. C. Caracterização morfo-anatômica e genética de quatro espécies invasoras de Commelina $\mathbf{L}$. 110 p. Tese (Doutorado em Botânica) - Universidade Estadual Paulista, Botucatu, 2001.

SILVA, J. R. V. Controle químico e deposição da calda de pulverização em dois estágios de desenvolvimento de plantas de Typha subulata Crespo \& Peres-Moreau. 74 p. Dissertação (Mestrado em Agricultura) - Universidade Estadual Paulista, Botucatu, 2003.
SOUZA, L. A. et al. Morfologia e anatomia vegetal: técnicas e práticas. Ponta Grossa: UEPG, 2005. 194 p.

SNEATH, P. H. A.; SOKAL, R. R. Numerical taxonomy. San Francisco: W. H. Freemam, 1973. 573 p.

TANAKA, R. H. et al. Ocorrência de plantas aquáticas nos reservatórios da Companhia Energética de São Paulo. Planta Daninha, v. 20, p. 101-111, 2002. Edição especial. 